Z. Klin. Chem. Klin. Biochem.

12. Jg. 1974 , S. $432-436$

\title{
Vergleichende Untersuchungen zur katalytischen Aktivität von Isoenzymen der alkalischen Phosphatase unter „konventionellen" und „optimierten“ Testbedingungen .,
}

\author{
Von J. Richter und J. Ohlen
}

Aus dem Institut für Klinische Chemie und Klinische Biochemie (Prof. Dr. A. Oberdorfer) und der II. Medizinischen Klinik und Poliklinik (Prof. Dr. H. Ley) des Klinikums rechts der Isar der Technischen Universität München

(Eingegangen am 6. Februar/13. Mai 1974)

Ein Vergleich der katalytischen Aktivitäten alkalischer Phosphatasen unter konventionellen und optimierten Testbedingungen in 600 Seren verschiedener Patienten und in hochgereinigten Isoenzym-Präparationen wurde durchgeführt. Für das Verhältnis der Aktivitätsbestimmungen mit beiden Testmethoden ergab sich keine konstante Beziehung, sondern ein variabler Faktor (f), der vom jeweiligen Isoenzymverteilungsmuster der alkalischen Phosphatasen abhängig war: gereinigte Placentaphosphatase $f=2,25$; Seren Schwangerer im letzten Trimenon $\mathrm{f}=3,62$; Seren mit hohen Knochenphosphataseaktivitäten $f=4,96$; Seren mit hohen Aktivitäten von hepatobiliären und intestinalen Phosphatasen $f=5,42$; gereinigte intestinale Phosphatase $f=6,29$.

\section{Comparative studies on the catalytic activity of isoenzymes of alkaline phosphatase, using "conventional" and "optimized" test conditions}

A comparison was made between the catalytic activities of alkaline phosphatases in the sera of 600 patients and in highly purified isoenzyme preparations when glycine or diethanolamine were used as buffer substance with $p$-nitrophenylphosphate as substrate. The enhancement of the catalytic activities brought about by the amino alcohol buffer as compared with glycine differed greatly depending on the origin of the alkaline phosphatases; this resulted in different conversion factors (f): purified placental phosphatases $f=2.25$; sera with predominantly placental phosphatases $f=3.62$; sera with predominantly bone phosphatases $f=4.96$; sera with predominantly hepatobiliary and intestinal phosphatases $f=5.42$; purified intestinal phosphatases $f=6.29$.

Bei der Bestimmung von Enzymaktivitäten wird die praktikable Annäherung an das Optimum als ,,optimierte Methode" bezeichnet (1). Für die alkalische Phosphatase (E. C. 3.1.3.1) erarbeiteten 1967 Rick (2) und Hausamen et al. (3) Bedingungen, die dieser Definition entsprechen. Während nachgewiesen werden konnte (4-6), daß der bei dieser Testoptimierung zu beobachtenden „Aktivierung" eine Transphosphorylierungsreaktion zugrunde liegt, ist bis heute nicht hinreichend untersucht worden, ob die konventionelle und die optimierte kinetische Methode (1) die Gesamtaktivität, d. h. die Summe der Aktivitäten von Knochen-, Leber/Gallenweg-, Dünndarm- und u. U. Placentaphosphatasen, in vergleichbarer Weise erfassen. Aufgabe der vorliegenden Arbeit war es, die katalytischen Aktivitäten von Isoenzymen der alkalischen Phosphatase unter konventionellen und optimierten Testbedingungen vergleichend zu untersuchen.

\section{Material und Methodik}

1. Es wurden die Seren von 600 verschiedenen Patienten (392 weibliche Patienten im Alter von 22 bis 74 Jahren; 208 männliche Patienten im Alter von 24 bis 72 Jahren) des ambulanten und stationären Krankengutes des Klinikums rechts der Isar der Technischen Universität München untersucht; die Gesamtaktivitäten der alkalischen Phosphatasen lagen in einem Bereich von 8 bis $150 \mathrm{U} / 1$ (konventioneller kinetischer Test, $25^{\circ} \mathrm{C}$ ) Unter den weiblichen Patienten befanden sich 35 gesunde Schwangere im letzten Trimenon. Die Auswahl der Seren erfolgte so, daß in den Aktivitätsbereichen bis 34 U/l (Kollektiv A) bzw. 34-150 U/1 (Kollektiv B) gleich große und etwa gleichmäßig verteilte Kollektive vorlagen. Seren mit höheren Aktivitäten, die eine Verdünnung notwendig gemacht hätten, wurden nicht für die Untersuchungen verwendet. Der Wert von $34 \mathrm{U} / \mathrm{l}$ stellt den oberen Grenzwert unseres lognormal (vergl. l.c. 3,7$)$ verteilten Normalkollektivs dar.

2. Dic Seren wurden 6 bis 8 Stunden nach der Blutabnahme in Portionen von $1,5 \mathrm{ml}$ innerhalb von 15 Minuten auf $-30^{\circ} \mathrm{C}$ tiefgefroren. Die Analyse der Seren von den Kollektiven A und $B$ wurde in 4 Serien zu je 150 Seren durchgeführt.

Die Bestimmung der Gesamtaktivität und der Aktivitäten der Isoenzyme der alkalischen Phosphatase erfolgte mit Hilfe von zwei Eppendorf-Enzymautomaten 5010 mit Enzymrechner 6450 gleichzeitig nach der konventionellen kinetischen und optimiert kinetischen Methode bei $25,0 \pm 0,2^{\circ} \mathrm{C}$ mit folgenden Endkonzentrationen für den konventionellen kinetischen Test: $50 \mathrm{mmol} / \mathrm{l}$ Glycinpuffer $\mathrm{pH} 10,5 ; 5,5 \mathrm{mmol} / \mathrm{l}$ Natrium- $p$-nitrophenylphosphat; $0,5 \mathrm{mmol} / 1$ Magnesiumchlorid; bzw. für den optimiert kinetischen Test: $1 \mathrm{~mol} / 1$ Diäthanolaminpuffer pH 9,$8 ; 10 \mathrm{mmol} / 1$ Natrium-p-nitrophenylphosphat; $0,5 \mathrm{mmol} / 1$ Magnesiumsulfat.

3. Die Bestimmung des Isoenzymverteilungsmusters der alkalischen Phosphatase wurde in allen Seren der Kollektive A und B durchgeführt. Die Isoenzymbestimmung erfolgte unmittelbar im Anschluß an die Bestimmungen der Gesamtaktivität unter konventionellen kinetischen Testbedingungen durch stereospezifische Hemmung mit $5 \mathrm{mmol} / \mathrm{l} L=$ Phenylalanin und 15 -MinutenHitzeinaktivierung bei $56,0 \pm 0,1^{\circ} \mathrm{C}$ in einem im einzelnen an 
anderer Stelle geschilderten kombinierten Verfahren $(8,9)$; Bestimmung eines Quotienten (Q-Wert), der das Verhältnis der Serumaktivitäten von hitzelabiler Knochenphosphatase zu den relativ hitzestabilen Leber/Gallenweg-Phosphatasen semiquantitativ erfaßt. Der Q-Wert ergibt sich aus der Aktivitätsbestimmung nach der Hitzeinaktivicrung mit $L$-Phenylalanin zu der vor der Hitzeinaktivierung mit $\boldsymbol{L}$-Phenylalanin, multipliziert mit dem Faktor 100. Normalbereich: $17,0 \pm 7,3(\bar{x} \pm 2 \mathrm{~s}$, $n=90$ ). Eine Ernicdrigung des. Q-Wertes weist auf eine Zunahme ossärer Phosphatasen hin, eine Erhöhung des Q-Wertes auf eine Zunahme hepatobiliärer Isoenzyme.

4. Zur Ermittlung von „Aktivierungsfaktoren" wurden vergleichend dic katalytischen Aktivitäten von hochgereinigten Isoenzympräparationen (Placenta- und Dünndarmphosphatasen) und von den nach unten angeführten Kriterien ausgewählten Serumkollektiven mit weitgehend isoliert erhöhten Isoenzymanteilen (Placenta-, ossäre und hepatobiliäre Phosphatasen) unter konventionellen kinetischen und optimiert kinetischen Testbedingungen bestimmt:

4.1. Hochgercinigte humane Placentaphosphatase (Boehringer, Mannheim) in $50 \mathrm{~g} / 1$ Albuminlösung: $\bar{x}$ (konv. kin. Test) = $124,7 \mathrm{U} / \mathrm{l}(\mathrm{n}=20)$.

4.2. 30 Seren von Schwangeren im 8.-10. Monat, in denen nach der Isocnzymbestimmung der Anteil von Placentaphosphatasen mehr als $70 \%$ der Gesamtaktivität betrug.

4.3. 45 Seren mit hohen Gesamtaktivitäten der alkalischen Phosphatase ( $>80 \mathrm{U} / 1$, konv. kin. Test) von Patienten mit Erkrankungen des Skelettsystems, die mit einer Steigerung der Osteoblastentätigkeit einhergingen. Nach der Isoenzymbestimmung waren mehr als $80 \%$ der Gesamtaktivität auf die Aktivität von Knochenphosphatasen zurückzuführen.

4.4. 60 Seren mit hohen Gesamtaktivitäten der alkalischen Phosphatase ( $>80 \mathrm{U} / 1$, konv. kin. Test) von Patienten mit hepatobiliären Erkrankungen. Nach der Isoenzy mbestimmung betrug der Anteil der Leber/Gallenweg-Phosphatasen mehr als 70\% der Gesamtaktivität.

4.5. Hochgereinigte Dünndarmphosphatase aus Kälberdarm (Analytical grade I, Boehringer, Mannheim) in $50 \mathrm{~g} / 1$ Albuminlösung: $\bar{x}$ (konv. kin. Test) $=21,7 \mathrm{U} / 1(n=20)$.

4.6. 120 Seren von gesunden Normalpersonen mit normalem Isoenzymvertcilungsmuster bei einer Gesamtaktivität der alkalischen Phosphatase von 7 bis $34 \mathrm{U} / \mathrm{l}$.

5. Die Kontrolle der Richtigkeit erfolgte durch Analysen des Kontrollserums „Enzatrol“" (Merz und Dade, München), welche zehn bis zwölf Stunden nach dem Auflösen durchgeführt wurden. Dic hierbei erhaltenen Ergebnisse wichen weniger als 5\% von dem Sollwert $a b$; damit konnte die Richtigkeit als gegeben betrachtet werden (10). Die Kontrolle der Präzision in den vier Scrien erfolgte durch Analy se von jeweils 20 Proben pro Serie von je zwei tiefgefroren aufbewahrten Human-Mischseren mit normaler und hoher Gesamtaktivität der alkalischen Phosphatase, die in den Probenketten des Enżymautomaten jeweils nach 8 Patientenseren analysiert wurden. Der Variationskoeffizient der Präzision in den Serien betrug für den konventionellen kinetischen Test $2,7-3,1 \%$ bei einer mittleren Aktivität von $\bar{x}=34,3 \mathrm{U} / 1$ und $1,6-2,1 \%$ bei einer mittleren Aktivität von $\overline{\mathrm{x}}=131,4 \mathrm{U} / 1$. Für die optimiert kinetische Methode betrug der Variationskoeffizient der Präzision in den Serien 1,5-2,9\% bei einer mittleren Aktivität von $\overline{\mathrm{x}}=181,8 \mathrm{U} / 1$ und $1,8-3,0 \%$ bei einer mittleren Aktivität von $\bar{x}=692,4$ U/1. Zur Ermittlung der unteren Nachweisgrenze wurde der Analysenblindwert durch Analyse von $50 \mathrm{~g} / 1$ Humanalbumin ,reinst" (Behring-Werke, Marburg) bestimmt. Die bei einer 20 fach-Bestimmung erhaltenen Mittelwerte $(\overline{\mathbf{x}})$ und Standardabweichungen (s) betrugen für die konventionelle kinetische Methode $\bar{x}=1,8 \mathrm{U} / 1, \mathrm{~s}=0,4 \mathrm{U} / 1$; für die optimiert kinetische Methode $\bar{x}=1,7 \mathrm{U} / 1, s=0,6 \mathrm{U} / 1$. Die nach Kaiser (11) hieraus resultierende untere Nachweisgrenze $\left(\overline{\mathbf{x}}_{\mathrm{BL}}+3 \mathrm{~s}_{\mathrm{BL}}\right)$ liegt demnach bei 3,0 U/1 bzw. 4,4 U/1.

6. Zur Signifikanzprüfung von Mittelwertsunterschicden für unverbundene Stichproben wurde nach vorheriger Testung auf Vorliegen einer Normalverteilung mit dem $x^{2}$-Anpassungs- test der t-Test angewendet. Für dic im Rahmen des Vergleichs der konventionellen kinetischen und optimiert kinetischen Bestimmungsmethode der alkalischen Phosphatase crmittelten katalytischen Aktivitäten wurden Regressionsanalysen durchgeführt und die Korrelationskoeffizienten (r) bestimmt (vergl. l.c. 12).

\section{Ergebnisse und Besprechung}

Nach den Untersuchungen von Rick (2) und Hausamen et al. (3) besitzt der bei der konventionellen Bestimmungsmethode der alkalischen Phosphatase von Bessey et al. (13) angegebene Glycin-NaOH-Puffer nur eine so begrenzte Pufferkapazität gegenüber dem Kohlendioxidgehalt der Luft und dem Proteingehalt des Serums, daß definierte Versuchsbedingungen nicht gewährleistet sind. Zur Optimierung und Standardisierung (14) der Bestimmung der alkalischen Phosphatase verwendeten diese Autoren 1,0 mol/1 Diäthanolamin-HCl-Puffer pH 9,8, der auch von der Kommission für Enzymdiagnostik und Standardisierung der Deutschen Gesellschaft für Klinische Chemie für die Bestimmung der alkalischen Phosphatase empfohlen wird (1). Der Mechanismus der bei dieser Optimierung zu beobachtenden ,Aktivierung“ der katalytischen Aktivitäten ist heute als Transphosphorylierungsreaktion weitgehend aufgeklärt (4-6).

1. Die Gesamtaktivitäten der alkalischen Phosphatase in den 600 verschiedenen Patientenseren wurden nach konventioneller kinetischer und optimiert kinetischer Methode gleichzeitig in Eppendorf-Analysenautomaten bestimmt. Die Korrelation der erhaltenen Analysenwerte ist in Abbildung 1 für Aktivitäten bis $34 \mathrm{U} / 1$ und in Abbildung 2 für Aktivitäten von 34 bis $150 \mathrm{U} / 1$ (konv. kin. Test) dargestellt. Unter der Annahme, daß bei der optimiert kinetischen Methode ein konstantes Vielfaches (k) der bei der konventionallen kinetischen Methode gemessenen Gesamtaktivität der alkalischen Phosphatase

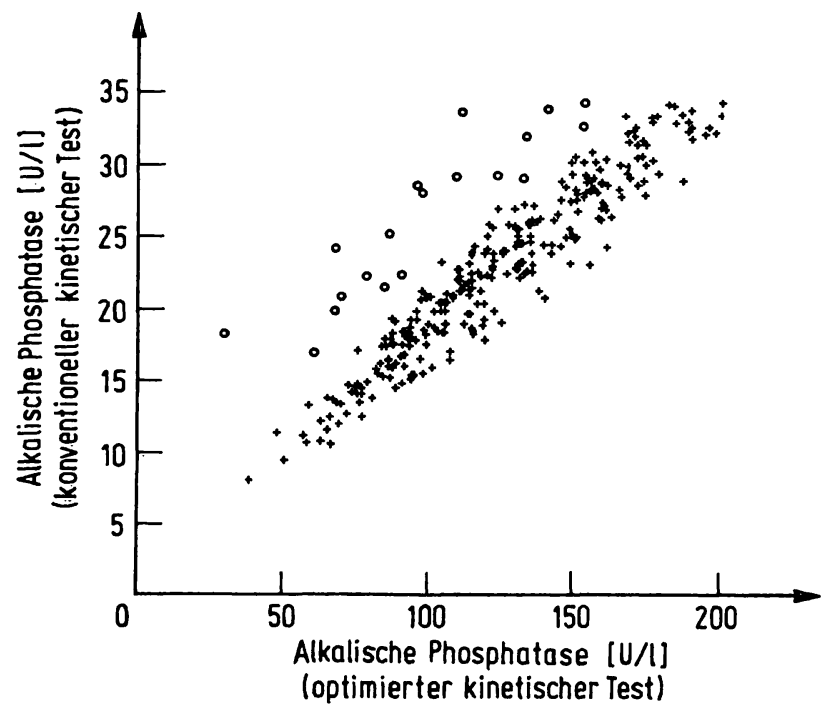

Abb. 1. Korrelation der nach konventioneller kinetischer bzw. optimiert kinetischer Methode bestimmten Gesamtaktivitäten (++) der alkalischen Phosphatase bis $34 \mathrm{U} / 1$ (konv. kin.Test). Die Seren von gesunden Schwangeren sind mit $\circ \circ$ gekennzeichnet. 


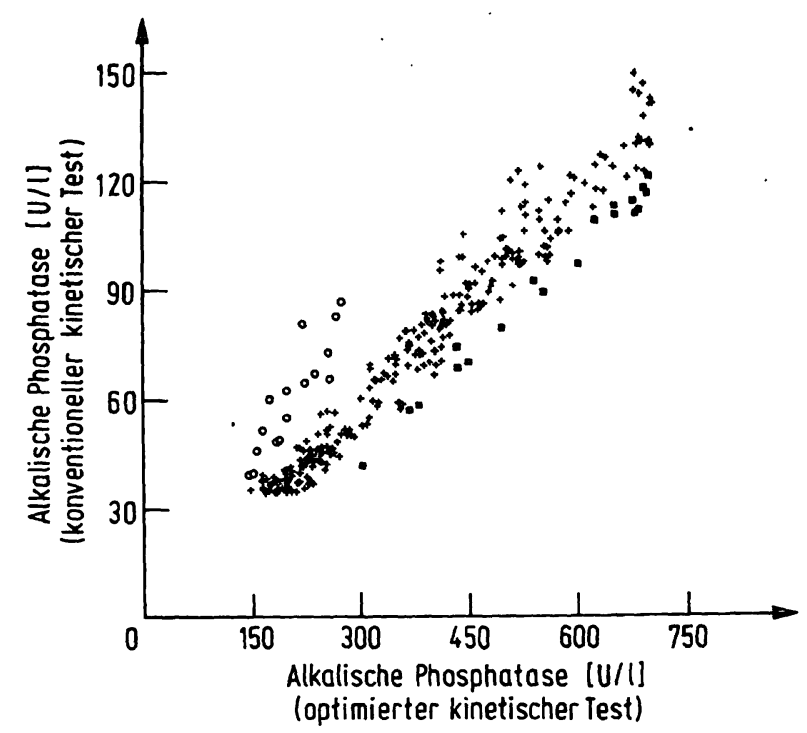

Abb. 2. Korrelation der nach konventioneller kinetischer bzw. optimiert kinetischer Methode bestimmten Gesamtaktivitäten $(++)$ der alkalischen Phosphatase von 34-150 U/1 (konv.kin.Test). Die Seren von gesunden Schwangeren sind mit $\circ$ o, die Seren von Patienten mit hepatobiliären Erkrankungen und hohen Anteilen von Dünndarm-Phosphatasen an der Gesamtaktivität sind mit a m gekennzeichnet.

(U/1) bestimmt würde, war die zu erwartende mathematische Beziehung: optimiert kinetische Methode (U/1) $=\mathrm{k} \times$ konventionelle kinetische Methode (U/1). In den Abbildungen 1 und 2 ließen sich trotz zum Teil guter Korrelationen $\left(r_{1}=+0,847\right.$ bzw. $\left.r_{2}=+0,909\right)$ zahlreiche Werte feststellen, die außerhalb der $95 \%$ Vertrauensgrenzen um die jeweiligen Regressionsgeraden lagen (vergl. l.c. 12).

2. Es wurden daher als mögliche diskriminierende Einflußgrößen die einzelnen Isoenzyme der alkalischen Phosphatase in den 600 Seren der Kollektive A und B bestimmt. Die Isoenzymbestimmung in den Seren des Kollektivs A ergab für 284 Seren eine Q-Wert-Bereichsspanne von 6 bis 34. In diesen Seren lagen bei $64 \%$ ein normales Isoenzymverteilungsmuster vor; bei $7 \%$ zeigte sich eine Vermehrung ossärer und bei $29 \%$ eine Vermehrung hepatobiliärer Isoenzyme. Die 16 Seren gesunder Schwangerer im letzten Trimenon zeigten mit Q-Werten von 52 bis 64 erwartungsgemäß $(15,16)$ einen hohen Anteil von Placentaphosphatasen an der Gesamtaktivität. Die Bestimmung des Isoenzymverteilungsmusters in der Gruppe mit erhöhten Aktivitäten der alkalischen Phosphatase (Kollektiv B) zeigte (Abb. 3), daß diese Gruppe aus drei Untergruppen bestand. Deutlich voneinander zu trennen waren zwei etwa gleichgroße Serumkollektive von Patienten mit ossären Erkrankungen und dadurch erhöhten Aktivitäten von Knochenphosphatasen bzw. von Patienten mit hepatobiliären Erkrankungen und dadurch bedingter Vermehrung der Leber/Gallenweg- und Dünndarmphosphatasen im Serum; ein drittes Kollektiv bestand aus 19 SchwangerenSeren mit hohem Anteil von Placentaphosphatasen. Diese Schwangeren-Seren zeigten bei der Bestimmung

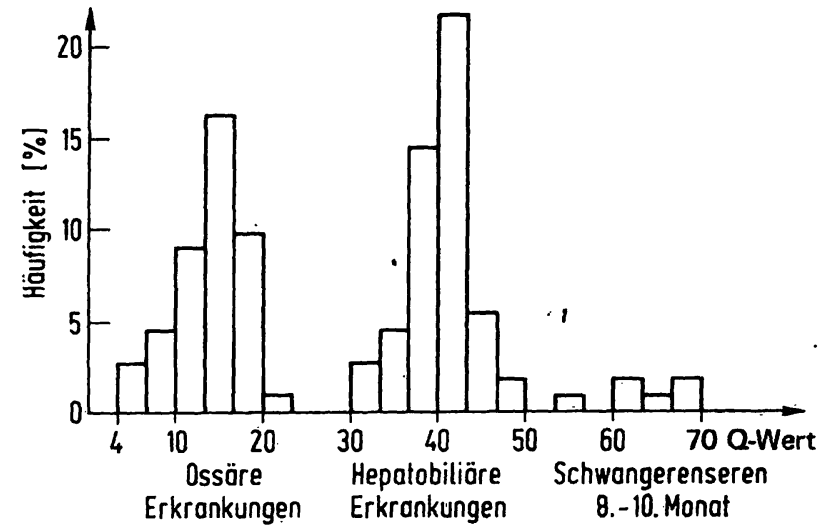

Abb. 3. Prozentuale Häufigkeit der Isoenzymverteilungen (Q-Werte, s. Methodik) in Seren mit Gesamtaktivitäten der alkalischen Phosphatase von 34-150 U/1 (konv.kin.Test).

der Gesamtaktivität der alkalischen Phosphatase ein gleichgerichtetes Verhalten zur Gesamt-Korrelation (Abb. 2) wie die Schwangeren-Seren in der Gruppe mit Aktivitäten innerhalb des Normalbereichs (Abb. 1). Eine dazu entgegengesetzte Abweichung von der Gesamt-Korrelation (Abb. 2) wiesen Wertepaare alkalischer Phosphataseaktivitäten von Seren auf, die von Patienten mit hepatobiliären Erkrankungen stammten und hohe Anteile von Dünndarmphosphatasen an der Gesamtaktivität besaßen.

Die vorstehenden Ergebnisse des Methodenvergleichs zeigten damit, daß die Annahme eines gleichen Aktivièrungsfaktors für alle Isoenzyme der alkalischen Phosphatase unter optimierten Testbedingungen nicht zutreffend war (vergl. 1. c. (17)).

3. In weiteren Untersuchungen wurde deshalb die Arbeitshypothese überprüft, nach der das Verhältnis von Aktivitätsbestimmungen mit beiden Testmethoden nicht mit einer Konstanten (k) zu beschreiben ist, sondern mit einem Faktor, der - abhängig vom Isoenzymverteilungsmuster - eine Funktion der unterschiedlichen „Aktivierungsfaktoren“ für die einzelnen Isoenzyme darstellt: $\mathrm{F}=f\left(\mathrm{f}_{1} \ldots \mathrm{f}_{\mathrm{n}} / \mathrm{AP}\right.$-Isoenzyme $)$. Dazu wurden die katalytischen Aktivitäten von hochgereinigten Isoenzympräparationen (Placenta- und Dünndarmphosphatasen) und von den unter Methodik beschriebenen ausgewählten Serumkollektiven mit weitgehend isoliert erhöhten Isoenzymanteilen (Placenta-, ossäre und hepátobiliäre Phosphatasen) unter konventionellen kinetischen und optimiert kinetischen Testbedingungen vergleichend bestimmt und die „Aktivierungsfaktoren“ für einzelne Isoenzyme bzw. Isoenzymgruppen ermittelt.

Bei der Untersuchung von hochgereinigten Placenta- und Dünndarmphosphatasen ergab sich im Vergleich zu den mit konventioneller kinetischer Testmethode ermittelten Werten eine deutlich unterschiedliche „Aktivierung“ der katalytischen Aktivitäten unter optimierten Testbedingungen (Tab. 1). Diese optimiert gemessenen Aktivitäten betrugen für die Placentaphosphatase das 2,25fache und die Dünndarmphosphatase das 6,29fache der mit der konventionellen Methode bestimmten Aktivitäten, 
Tab. 1. Angabe von Mittclwert und einfacher Standardabweichung $(\bar{x} \pm s)$ für die unter optimiert kinetischen Testbedingungen im Vergleich zur konventionellen kinetischen Methode ermittelten „Aktivierungsfaktoren" für isolierte Isoenzyme bzw. Gesamtaktivitäten der alkalischen Phosphatase in ausgewählten Sereri von Schwangeren (8.-10. Monat), Patienten mit ossären und hepatobiliären Erkrankungen sowie von Normalpersonen.

\begin{tabular}{lrl}
\hline Untersuchtes Matcrial & $\mathrm{n}$ & $\begin{array}{l}\text {,Aktivierungs- } \\
\text { faktoren“ } \\
(\overline{\mathbf{x}} \pm s)\end{array}$ \\
\hline $\begin{array}{l}\text { Hochgereinigtc humanc } \\
\text { Placentaphosphatasen }\end{array}$ & 20 & $2,25 \pm 0,30$ \\
$\begin{array}{l}\text { Schwangerenseren 8.- 10. Monat } \\
\text { Seren von Patienten mit ossären }\end{array}$ & 30 & $3,62 \pm 0,42$ \\
$\begin{array}{l}\text { Erkrankungen } \\
\text { Normalpersonen }\end{array}$ & 45 & $4,96 \pm 0,24$ \\
$\begin{array}{l}\text { Seren von Patienten mit hepatobiliären } \\
\text { Erkrankungen }\end{array}$ & 60 & $5,42 \pm 0,39$ \\
Hochgereinigtc Dünndarmphosphatasen & 20 & $6,29 \pm 0,26$ \\
\hline
\end{tabular}

d. h. daß die katalytische Aktivität der Dünndarmphosphatase unter optimierten Testbedingungen nahezu dreimal höher war als die der Placentaphosphatase. Entsprechend der geringeren Aktivierbarkeit der Placentaphosphatase unter optimierten Testbedingungen fand sich für die Werte der Gesamtaktivität in Seren Schwangerer im letzten Trimenon beim Vergleich beider Testmethoden ein Faktor von 3,62 (Faktoren um den Wert 3,2 finden sich auch bei handelsüblichen Kontrollseren, bei denen als ,Alkalische Phosphatase"-Anteil Placentaphosphatasen im Lyophilisat enthalten sind). In den Seren von Patienten mit starker Vermehrung hepatobiliärer Isoenzyme wurden die Gesamtaktivitäten der alkalischen Phosphatase unter optimierten Testbedingungen um den Faktor 5,42 höher gemessen als mit der konventionellen Methode; für Seren von Patienten mit eindeutiger Erhöhung von Knochenphosphatasen betrug der „Aktivierungsfaktor“ nur 4,96 (Tab. 1). Dieser, wenn auch geringe, Unterschied war statistisch signifikant $(p \leqslant 0,01)$.
Haije (17) fand eine ähnlich geringe Abweichung der katalytischen Aktivitäten von Knochen- und Leber/ Gallenweg-Phosphatasen beim Vergleich der Umsetzungsgeschwindigkeiten von $p$-Nitrophenylphosphat in Glycin-bzw. 2-Amino-2-methyl-1-propanol-Puffer.

4. Die differierenden „Aktivierungsfaktoren“ zeigen, daß die Arbeitshypothese, die das Verhältnis von Aktivitätsbestimmungen mit beiden Methoden als Funktion der unterschiedlichen „Aktivierungsfaktoren“ für die einzelnen Isoenzyme der alkalischen Phosphatase definierte, zutreffend war. Ein einfaches Beispiel für die Richtigkeit dieser mathematischen Beziehung: Nach unseren früheren Untersuchungen $(8,9)$ setzt sich die Gesamtaktivität der alkalischen Phosphatase im Serum normaler gesunder Erwachsener zu etwa 70\% aus Knochen-, zu etwa $20 \%$ aus Dünndarm- und zu etwa $10 \%$ aus hepatobiliären Phosphatase-Aktivitäten zusammen. Mittelt man diesen Anteilen entsprechend die gefundenen ,Aktivierungsfaktoren“ $(70 \times 4,96+20 \times 6,29+$ $+10 \times 5,42$ dividiert durch 100 ), so erhält man einen Faktor von 5,27. Dieser rechnerisch ermittelte Wert für Seren mit normalem Isoenzymverteilungsmuster deckt sich gut mit jenem ,Aktivierungsfaktor" von 5,22, der sich als mittlerer Faktor aus vergleichenden Aktivitätsbestimmungen in Seren von 120 Normalpersonen ergab (Tab. 1).

5. Zusammenfassend kann festgestellt werden, daß die katalytischen Aktivitäten der Isoenzyme der alkalischen Phosphatase unter konventionellen kinetischen und optimiert kinetischen Testbedingungen unterschiedlich erfaßt werden. Ohne auf die damit verbundene Problematik der Richtigkeit irgendeiner Aktivitätsbestimmung der alkalischen Phosphatase eingehen zu können, ergab sich aus den vorliegenden Untersuchungen für das Verhältnis der Aktivitätsbestimmungen mit beiden Testmethoden keine konstante Beziehung, sondern ein variabler Faktor, der vom jeweiligen Isoenzymverteilungsmuster der alkalischen Phosphatase im Serum abhängig war.

\section{Literatur}

1. Empfehlungen der Deutschen Gesellschaft für Klinische Chemie (1970), diese Z. 8, 658-659.

2. Rick, W., Hausamen, T.-U. (1967), Ergeb. Labor. Med. 3, 86-90.

3. Hausamen, T.-U., Helger, R., Rick, W. \& Gross, W. (1967), Clin. Chim. Acta 15, 241-245.

4. Amador, E. A. \& Urban, J. (1972), Amer. J. Clin. Pathol. 57, 167-172.

5. McComb, R. B. \& Bowers, G. N. (1972), Clin. Chem. 18, 97-104.

6. Zech, R., Grote, M., Zircher, K.\& Schlaeger, R. (1973), diese Z. 11, 461 -464 .

7. Wootton, I. D. P. \& King, E. J. (1953), Lancet I, 470-471.

8. Ohlen, J. \& Richter, J. (1971), Deut. Med. Wochenschr. 96, 343-345.

9. Ohlen, J., Pause, H. \& Richter, J. (1971), Europ. J. Clin. Invest. $1,445-451$.

10. Stamm, D. (1972), Qualitätskontrolle klinisch-chemischer Analysen, Georg Thieme Verlag, Stuttgart.

11. Kaiser, H. (1966), Z. Anal. Chem. 216, 80-84.

12. Sachs, L. (1972), Statistische Auswertungsmethoden, 3. Aufl., Springer-Verlag Berlin-Heidelberg-New York.

13. Bessey, O. A., Lowry, O. H. \& Brock, M. J. (1946), J. Biol Chem. 164, 321-328. 
14. Report of the Commission on Enzymes of the International Union of Biochemistry, IUB Symposia, Series 20, Pergamon Press, Oxford (1961).

15. Sussman, H. H., Bowman, M. \& Lewis, J. L. (1968); Nature (Lond.) 218, 359-360.
16. Posen, S., Cornish, C. J., Horne, M. \& Saini, P. K. (1969), Ann. N. Y. Acad. Sci. 166, 733-744.

17. Haije, W. G. (1973), Clin. Chim. Acta 48, 23-26.

Priv.-Doz. Dr. J. Richter

Inst. f. Klin. Chem. u. Klin. Biochemie d. TU

Dr. J. Ohlen

II. Med. Klin. u. Poliklin. d. TU

8 München 80

Ismaningerstr. 22

\section{Notice of the IUB Commission of Editors of Biochemical Journals}

\section{Revision of enzyme nomenclature}

Following the publication of Enzyme Nomenclature Recommendations 1972*, the Commission on Biochemical Nomenclature has dedided to maintain a continuing review of the Enzyme List contained in it and to publish supplements and corrections from time to time. It is hoped that such a supplement can be published annually in a biochemical journal, and that about every third year these can be combined into a small separate volume.

The Commission invites all members of the biochemical community to assist in this task, by drawing attention to entries in the existing list which appear to be incorrert, or to new enzymes which should be includea. All such information should be sert to

Professor Edwin C. Webb, J. D. Story Building, University of Queensland, St. Lucia, 4067 Australia.
For new enzymes as much of the following information as possible should be included:

Reaction catalysed; a brief note on specificity; suggested classification sub-group; suggested name; source of enzyme; reference.

A pro-forma is available from the Editorial Office for ease in setting out these particulars.

In the case of a correction of an existing entry, correspondents should set out clearly what is wrong with the material published.

For inclusion in the first annual supplement, material must be received in Brisbane not later than

December 31st, 1974.

Edwin C. Webb

\footnotetext{
* IUPAC-IUB Commission on Biochemical Nomenclature (1973) Enzyme Nomenclature, Recommendations, 1972, Elsevier, Amsterdam.
}

\section{Preisverleihung}

Herr Professor Dr. Dr. Dankwart Stamm, Leiter der Abteilung für Klinische Chemie des Max-Planck-Institutes für Psychiatrie in München, wurde für seine Arbeiten auf dem Gebiet der Qualitätskontrolle in der Klinischen Chemie durch die Verleihung des

\section{Dade Geneva Symposium-Preises}

ausgezeichnet. 